\title{
Preliminary Investigation of Risk Factors Causing Dyskinesias in Parkinson's Disease in South Africa
}

\author{
Razia Gaida and Ilse Truter \\ Drug Utilisation Research Unit (DURU), Department of Pharmacy, Nelson Mandela Metropolitan University (NMMU), Port \\ Elizabeth, South Africa \\ *For correspondence: Email: ilse.truter@nmmu.ac.za; Tel: +2741-5042131; Fax: +2741-5042744
}

\begin{abstract}
Purpose: To determine the risk factors involved in the onset of dyskinesias in patients suffering from Parkinson's disease in South Africa.

Methods: A questionnaire survey and medical record review were conducted. A total of 43 patients with Parkinson's disease in two metropolitan areas were included in the study.

Results: The number of patients with dyskinesias indicated by medical records $(n=10)$ was less than the number of patients with self-reported dyskinesias $(n=13)$. According to the patient interviews, the mean prescribed daily dose (PDD) of levodopa was $750 \pm 452 \mathrm{mg}$. Twelve patients had been using levodopa for $>10$ years. Mean PDD of levodopa according to the medical records was $809 \pm 514 \mathrm{mg}$, with dyskinetic patients receiving a higher average PDD of $870 \pm 590 \mathrm{mg}$. Half of the patients with dyskinesias were diagnosed more than 10 years ago. Patients with dyskinesias were diagnosed with Parkinson's disease at a younger age (11.63\% dyskinetic patients were diagnosed between 40 and 60 years), and experienced longer disease duration. There were no gender differences $(p=0.80)$ in the incidence of dyskinesias.

Conclusion: Higher doses of levodopa, longer disease duration and a longer duration of levodopa therapy were risk factors for dyskinesias. Further studies are needed on the incidence of dyskinesias.
\end{abstract}

Keywords: Parkinson's disease, Dyskinesias, Levodopa; Drug utilisation South Africa

Tropical Journal of Pharmaceutical Research is indexed by Science Citation Index (SciSearch), Scopus, International Pharmaceutical Abstract, Chemical Abstracts, Embase, Index Copernicus, EBSCO, African Index Medicus, JournalSeek, Journal Citation Reports/Science Edition, Directory of Open Access Journals (DOAJ), African Journal Online, Bioline International, Open-J-Gate and Pharmacy Abstracts

\section{INTRODUCTION}

Patients with Parkinson's disease typically present with tremor, rigidity and bradykinesia [13]. Levodopa is regarded as the 'gold standard' symptomatic treatment for Parkinson's disease, however, after 4 - 5 years of treatment, levodopa efficacy tends to decline $[1,4]$. Patients on longterm treatment with levodopa experience motor fluctuations and dyskinesias (impaired voluntary movements which result in fragmented movement which only cease during sleep) [5]. Dyskinesias, also termed levodopa-induced dyskinesias (LIDs), are a major limitation in the therapy of Parkinson's disease [5]. Yet, the ELLDOPA (Early versus Later Levodopa in Parkinson's Disease) study did not find evidence to suggest that levodopa accelerate the worsening of Parkinson's disease [6].

LIDs are more commonly associated with high daily doses of levodopa, ranging from $400 \mathrm{mg}$ to $600 \mathrm{mg}$ daily $[7,8]$. Also, younger patients experience dyskinesias more frequently than older patients. In a study of 50 patients with disease duration of 10 years and longer, $56 \%$ developed drug-induced dyskinesias within the first 10 years [9], and incidence was related to 
disease severity. Further studies noted that patients in later stages of the disease were more likely to develop dyskinesias earlier than patients in earlier stages [10], and it was demonstrated that patients between 40 and 49 years and those between 50 and 59 years presented with $40 \%$ and $53 \%$ incidence respectively, whereas patients over 60 years presented lower than 26 $\%$ incidence [11].

A further factor when determining a patient's risk for the development of dyskinesias is the levodopa dose per kilogram [12,13]. Not all patients on similar levodopa doses develop dyskinesias as $60 \%$ of patients remain free of any dyskinesia after four to six years of therapy [13]. A possible explanation for this is the body mass of the patient [13]. This was also seen as the reason for women experiencing dyskinesias more frequently than men, since women weighed less and were receiving higher levodopa doses per kilogram [13].

A major controversy in Parkinson's disease therapy is whether to withhold levodopa as the initial therapy in order to delay the onset of dyskinesias or to provide the patient with this 'gold standard' therapy in order to obtain maximum symptomatic control. There has not been any previous study identified on the risk factors for the development of dyskinesias in South Africa. The primary aim of this study was therefore to determine the risk factors involved in the onset of dyskinesias in patients suffering from Parkinson's disease in South Africa.

\section{EXPERIMENTAL}

\section{Study design}

A questionnaire was developed to determine the presence of dyskinesias in patients with Parkinson's disease as well as possible risk factors such as the date of diagnosis, disease duration and levodopa dose. The information obtained included the age of onset of Parkinson's disease, when levodopa therapy was initiated relative to the date of diagnosis, the use of other medications, even if not antiparkinsonian medication, if and when the emergence of dyskinesias occurred, the severity of the dyskinesias as well as the risk factors involved in the emergence of dyskinesias. A retrospective review of the medical records of participating patients was also conducted to obtain an objective view of the patients' conditions. A total of 43 patients were included in the study.
Patients were sourced from two hospitals in the Western Cape Province which are both public sector institutions. The neurology outpatient clinic at Hospital A operated on Tuesday afternoons and Thursday mornings. This hospital is the training hospital for the medical school of a university in the Western Cape. The patient population at Hospital A was too small to obtain the desired number of patients. Hospital $B$ was chosen as it is another public sector hospital in the Western Cape with a neurologist and it runs a specialised Parkinson's disease clinic every two weeks. This hospital is the training hospital for the medical school at another university in the Western Cape. The Parkinson's disease support group in Port Elizabeth was also used as a source of patients as the specialised clinics at Hospital A and Hospital B were not yielding as many patients as originally anticipated. Port Elizabeth and Cape Town were therefore included as study sites. Data collection took place from May 2011 until September 2012.

A pilot study was conducted to test whether the questions were unambiguous and whether patients would understand the questions, as well as to determine how long it will take to complete the questionnaire. Five patients from Hospital $A$ were included in the pilot study. Their results were not included in the final study. Patient files were scanned in numerical order at both hospitals and patients older than 50 years and who had been on levodopa therapy for more than one year were selected. The final questionnaire was verbally administered to patients diagnosed with Parkinson's disease to ensure $100 \%$ response rate. The survey took place in a comfortable, confidential environment where they were able to openly communicate. An unused office in the clinic was available for the interviews. Each patient was asked whether they want to stay where they were or want to move to the office. Some chose to stay where they were, whilst others preferred to be interviewed in the office.

\section{Ethical considerations}

Ethical approval was obtained from the Research Ethics Committees (Human) of the Nelson Mandela Metropolitan University (ethics clearance reference number: H11-HEA-PHA001), the University of Cape Town and Stellenbosch University. The hospital management of both public sector hospitals provided permission to interview patients and access to medical records. Informed consent was requested from patients prior to participation in the study and confidentiality was maintained at all times. 


\section{Data analysis}

A retrospective review of the participating patients' medical records was conducted using a data sheet which aimed to obtain information concerning the date of diagnosis, current disease severity, initial therapy prescribed, date of levodopa initiation, initial dose of levodopa, current levodopa regimen, other antiparkinsonian medications the patient was receiving, incidence of dyskinesias and latency to onset of dyskinesias. The data were captured using Microsoft Excel $囚$.

Microsoft Excel $®$ and Statistica ${ }^{\circledR}$ were used for statistical analyses. Basic descriptive and inferential statistics were calculated. The results are expressed as mean \pm standard deviation. Statistical significance was determined by Chisquare tests for the frequency of dyskinesias according to the duration of levodopa therapy. A value of $p<0.05$ was considered significant.

\section{RESULTS}

\section{Demographic information of patients}

Of the 43 patients interviewed, 23 were males (53.49 \%). Parkinson's disease was therefore more commonly found in male patients than female patients in this sample with a female to male ratio of $1: 1.15$. The majority of participants were coloured ( $n=22 ; 51.16 \%)$ with the second largest ethnic group being white $(n=16 ; 37.21$
$\%$ ). Most coloured patients were identified in the public sector while the white group was made up mostly of private sector patients $(68.75 \%)$. The majority of the patients were from Hospital $B(n=$ $22 ; 51.16 \%)$, with the support group in Port Elizabeth being the second largest pool of patients ( $\mathrm{n}=12 ; 27.91 \%$ ) and Hospital $\mathrm{A}$ provided nine patients $(20.93 \%)$ of the total 43 .

\section{Results from the patient interviews}

There were 14 patients aged between 71 and 75 years $(32.56 \%)$ who constituted the largest age group overall. Male patients were found to be older than female patients. Of the 23 male patients, $73.91 \%$ were 66 years or older as compared to females where only $55.00 \%$ were 66 years or older. The majority of patients were diagnosed with Parkinson's disease between five and 10 years before the onset of the study $(n=$ $15 ; 34.88 \%$ ) (Figure 1). Six patients diagnosed more than 10 years before the onset of the study were over the age of 70 years.

The average Prescribed Daily Dose (PDD) of levodopa was $750 \mathrm{mg} \pm 452 \mathrm{mg}$. Fourteen patients were using levodopa for two to four years and 12 were using levodopa for more than 10 years. Fifteen patients were using levodopa for the entire duration of their disease. The majority of patients ( $n=27 ; 62.79 \%$ ) were using the levodopa/carbidopa 100 / 25 mg formulation.

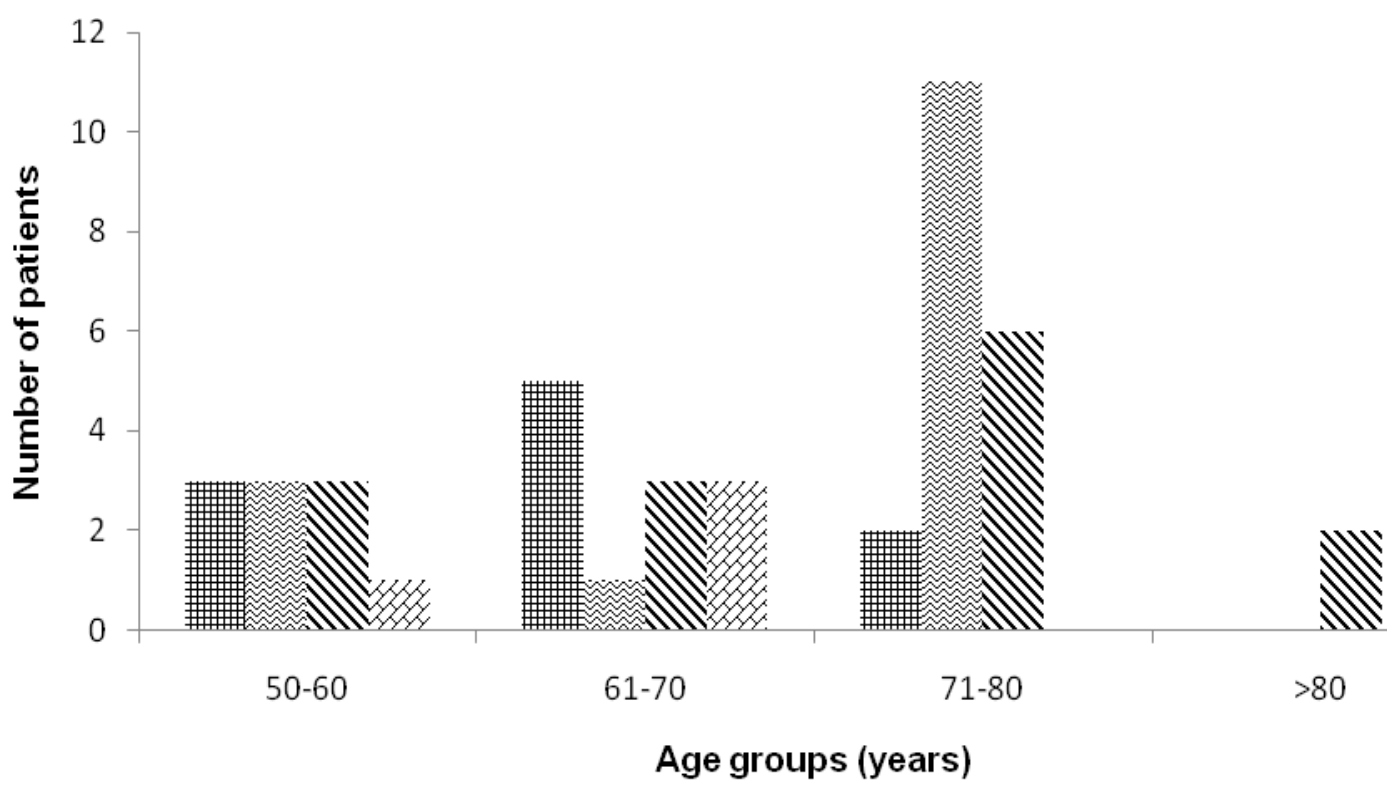

\# Less than 5 years $\approx$ Between 5 and 10 years $\quad$ More than 10 years 2 Unsure

Figure 1: Disease duration of patients according to 10 year age groups $(n=43)$ 
Dyskinetic patients received a higher average PDD of $870 \pm 590 \mathrm{mg}$. There were some patients who admitted to altering their doses to obtain, and what they felt was better symptomatic relief. Thirty-six (83.72\%) patients were also using other medication such as antihypertensive drugs, hypoglycaemic agents, lipid lowering agents and antidepressants. Patients with longer disease duration were receiving higher daily doses of levodopa.

A positive incidence of dyskinesias was reported by 13 patients $(30.23 \%)$. When applying finer age categories to the study population, it was seen that the majority of these patients were in the age groups 56 to 60 years $(n=4)$ and 71 to 75 years $(n=4)$. Of those reporting a positive incidence, seven were males and six were females $(p=0.98)$. Five patients were diagnosed with Parkinson's disease more than 10 years ago $(38.46 \%)$. Table 1 indicates the frequency of dyskinesias according to disease duration. The chi-square test yielded nonsignificant results and a p-value of 0.95 was found. Table 2 indicates the frequency of dyskinesias according to the duration of levodopa therapy.

Statistical analysis showed that there were some differences. However, due to the small sample size, the chi-square test yielded a non-significant result with a $p$-value of 0.4138 . There were five patients who reported being on levodopa therapy for just two to four years. Of these, three were diagnosed less than five years ago. Four patients were reported to be using levodopa for more than 10 years.

Table 2: Frequency of dyskinesias in patients according to duration of levodopa therapy $(n=13)$

\begin{tabular}{lcc}
\hline \multirow{2}{*}{$\begin{array}{l}\text { Levodopa } \\
\text { duration }\end{array}$} & \multicolumn{2}{c}{ Dyskinesia } \\
\cline { 2 - 3 } 2-4 years & 5 & No/Unsure \\
5-7 years & 1 & 9 \\
8-10 years & 3 & 6 \\
11+ years & 4 & 3 \\
Not sure & 0 & 8 \\
Total & $\mathbf{1 3}$ & 4 \\
\hline
\end{tabular}

\section{Results from the medical record review}

According to the medical records, most of the patients were diagnosed between the ages of 56 to 60 years ( $\mathrm{n}=9 ; 20.93 \%$ ) and 61 to 65 years ( $n=9 ; 20.93 \%$ ). The medical records showed that $44.19 \%$ of patients were diagnosed between five and 10 years before the onset of the study, $23.26 \%$ were diagnosed less than five years, and $27.91 \%$ more than 10 years.

The average PDD of levodopa was $809 \mathrm{mg} \pm$ $514 \mathrm{mg}$ with a range of $100 \mathrm{mg}$ to $2400 \mathrm{mg}$. The most commonly prescribed average PDD of levodopa was $300 \mathrm{mg}(\mathrm{n}=7 ; 16.28 \%)$. Males were receiving higher doses of levodopa with a mean of $858 \mathrm{mg} \pm 490 \mathrm{mg}$. Females were using a mean dose of $642.50 \mathrm{mg} \pm 400.75 \mathrm{mg}$. Higher doses of levodopa were associated with longer disease duration.

Eight patients (18.60 \%) were prescribed amantadine. Eleven patients $(25.81 \%)$ were prescribed an anticholinergic agent. Six patients (13.95 \%) were prescribed benzhexol, two patients $(4.65 \%)$ were prescribed biperidine and five patients $(11.63 \%)$ were prescribed orphenadrine. Of the two patients prescribed biperidine neither were continued on the medication. Only two patients $(4.65 \%)$ were prescribed entacapone. One patient discontinued the medication.

Twenty patients were prescribed a dopamine agonist $(46.51 \%)$. Five patients $(11.63 \%)$ were prescribed bromocriptine, six patients (13.95\%) were prescribed pramipexole and 15 patients $(34.88 \%)$ were prescribed ropinirole. There were also two patients $(4.65 \%)$ prescribed pergolide, however, both were discontinued after a period of six and nine years, respectively.

Four patients $(9.30 \%)$ were prescribed MAO-B inhibitors. Of these, rasagiline was the more popular drug with three patients out of the four being prescribed as such. However, only one of these patients continued therapy on a MAO-B inhibitor. Domperidone and metoclopramide

Table 1: Frequency of dyskinesias in patients according to disease duration $(n=13)$

\begin{tabular}{lcc}
\hline $\begin{array}{l}\text { Disease duration as from date of } \\
\text { diagnosis }\end{array}$ & \multicolumn{3}{c}{ Dyskinesias } \\
\cline { 2 - 3 } & Yes & No/Unsure \\
\hline 5 years & 3 & 7 \\
11+ years & 4 & 11 \\
Not yure & 5 & 9 \\
Total & 1 & 3 \\
\hline
\end{tabular}


were prescribed to two and one patient, respectively. Two patients received donepezil, an anticholinesterase, and one patient received memantine, an NMDA-receptor antagonist and a derivative of amantadine.

According to the medical records, 10 patients were diagnosed with dyskinesias, of which half were males. Patients experiencing dyskinesias were diagnosed with Parkinson's disease earlier than non-dyskinetic patients.

Half of the patients positively diagnosed with dyskinesias were diagnosed more than 10 years ago. Table 3 indicates the initial and current PDDs of levodopa according to the medical records of dyskinetic patients.

In the instances where information was not available, patients had been referred from one hospital to another and information was absent from the records. When comparing the initial and current doses, it was seen that the doses were increased in only three cases.

In the instances where the dose has been decreased, it is normally done to provide control of dyskinesias. The majority of patients with dyskinesias were on levodopa therapy for less than five years $(n=4 ; 40 \%)$. Four patients did not have the information available in their medical records.

\section{DISCUSSION}

The number of patients with dyskinesias as indicated by the medical records $(n=10)$ was less than the number of patients with selfreported dyskinesias $(n=13)$. A reason could be that patients did perhaps not fully understand what a dyskinesia was and answered positively. The study showed that patients with dyskinesias had experienced longer disease duration. This was in line with the results of other studies which state that there is a positive relationship between disease duration and the risk of dyskinesias $[7,14]$. Due to the small number of patients, when combining them into age groups 40 to 60 years, 61 to 70 years and 70 years and older, it was seen that five patients diagnosed between the ages of 40 to 60 years experienced dyskinesias. The incidence then decreased to two patients who were diagnosed between the ages of 61 to 70 years and one patient experiencing dyskinesias who was diagnosed at 70 years or older. This finding was similar to other studies found $[11,15]$.

Dyskinetic patients received a higher average levodopa dose of $870 \mathrm{mg} \pm 590 \mathrm{mg}$ compared to the average of $790.91 \mathrm{mg} \pm 497.88 \mathrm{mg}$ received by non-dyskinetic patients and the overall average of $809 \mathrm{mg} \pm 514 \mathrm{mg}$ as according to the medical records.

Table 3: Initial and current PDDs of levodopa according to medical records of dyskinetic patients

\begin{tabular}{lll}
\hline Patient & Initial PDD & Current PDD \\
\hline Patient 1 & $300 \mathrm{mg}$ & $2000 \mathrm{mg}$ \\
Patient 2 & Information not available & $1000 \mathrm{mg}$ \\
Patient 8 & $600 \mathrm{mg}$ & $1000 \mathrm{mg}$ \\
Patient 11 & $150 \mathrm{mg}$ & $100 \mathrm{mg}$ \\
Patient 16 & $750 \mathrm{mg}$ & $600 \mathrm{mg}$ \\
Patient 23 & $900 \mathrm{mg}$ & $800 \mathrm{mg}$ \\
Patient 26 & $600 \mathrm{mg}$ & $1500 \mathrm{mg}$ \\
Patient 27 & Information not available & $250 \mathrm{mg}$ \\
Patient 36 & $450 \mathrm{mg}$ & $450 \mathrm{mg}$ \\
Patient 43 & Information not available & $1000 \mathrm{mg}$ \\
\hline
\end{tabular}


When comparing the doses of levodopa being used at the time of the study, the patient interviews and medical records were almost identical. This indicates that most patients adhered to their prescriptions, although some patients admitted to altering their doses to obtain better symptomatic relief.

Eight patients were prescribed amantadine. According to the World Health Organisation (WHO) the Defined Daily Dose (DDD) is the assumed average maintenance dose per day for a drug used for its primary indication in adults [16]. The DDD of amantadine is $200 \mathrm{mg}$, but two patients were receiving higher doses than this (300 mg). Domperidone and metoclopramide were prescribed to two patients. Both these agents are dopamine antagonists used to increase gastric motility [17]. However, in Parkinson's disease neurologists may administer domperidone, concurrently with levodopa, in order to reduce the incidence of peripheral side effects caused by the dopaminergic drug. Domperidone does not cross the blood brain barrier [17]. According to Daily Drug Use [18], metoclopramide reduces the effects of levodopa and concurrent use should be avoided. Two patients received donepezil and memantine. The primary indication for these drugs in Parkinson's disease is dementia [17].

A Turkish study [7] showed that patients with an average initial dose of levodopa of $291 \mathrm{mg}$ daily resulted in dyskinesias. Another study showed that dyskinesias were seen in patients receiving average levodopa doses of $338 \mathrm{mg}$ daily [19]. The current study showed that eight patients were receiving daily doses higher than this. Not all patients developed dyskinesias, even though the dose of levodopa was high. This can be seen by patients 14 and 33 who both received daily doses of levodopa of $2100 \mathrm{mg}$ and $2400 \mathrm{mg}$, respectively. Information concerning the patients' current mass was not available from either the patients or the medical records. The levodopa dose per kilogram could therefore not be calculated.

\section{Limitations of the study}

This study formed a preliminary investigation of the risk factors causing dyskinesias in South Africa. Data collection took place in Cape Town and Port Elizabeth and therefore the study is not a reflection of the South African population as a whole. The study focused on levodopa causing dyskinesias, however, there is the possibility that other classes of drugs used for the treatment of
Parkinson's disease and used by some of the patients may have played a role in the development of the dyskinesias. Finally, the ethnic division may have been biased as most of the data collection took place in the Western Cape, which holds the highest proportion of coloureds in South Africa. Further investigation is warranted in terms of the demographical distribution as these results may not be an accurate representation of Parkinson's disease in the South African population amongst the different ethnic groups.

\section{CONCLUSION}

The primary aim of this study was to determine the risk factors involved in the onset of dyskinesias in patients suffering from Parkinson's disease in South Africa. The findings indicated that longer disease duration, higher levodopa doses and a longer duration of levodopa therapy were risk factors in the development of dyskinesias. Parkinson's disease is an underrecognised condition in South Africa. It is recommended that a more comprehensive questionnaire survey be conducted to determine whether the incidence of Parkinson's disease in South Africa differs between racial or cultural groups. Further investigation into the effectiveness of the various drug classes used in the treatment of Parkinson's disease and comparing these classes is warranted in order to establish more concrete treatment guidelines. Finally, it is recommended that weight-based dosing of levodopa should be taken into account and that its effect on the incidence of dyskinesias, particularly in female patients, be investigated.

\section{ACKNOWLEDGEMENT}

The Nelson Mandela Metropolitan University (NMMU) and the National Research Foundation (NRF) for financial assistance in the form of bursaries to conduct this study. Any opinion, findings and conclusions or recommendations expressed in this paper are those of the authors and therefore the NRF does not accept any liability in regard thereto.

\section{REFERENCES}

1. Singh N, Pillay $V$, Choonara YE. Advances in the treatment of Parkinson's disease. Prog Neurobiol 2007; 81(1): 29-44.

Trop J Pharm Res, August 2014; 13(8): 1358 
2. Tolosa E, Wenning G, Poewe W. The diagnosis of Parkinson's disease. Lancet Neurol 2006; 5(1): 7586.

3. Obeso JA, Olanow CW, Nutt JG. Levodopa motor complications in Parkinson's disease. Trends Neurosci 2000; 23(10): 2-6.

4. Stern M. The early treatment of Parkinson's disease: levodopa, dopamine agonists, or both. Parkinsonism \& Related Disorders 2001; 7: 27-33.

5. Prashanth LK, Fox S, Meissner WG. L-dopa induced dyskinesia - clinical presentation, genetics, and treatment. Int Rev Neurobiol 2001; 98(2011): 31-54.

6. Fahn S. Parkinson's disease, the effect of levodopa, and the ELLDOPA trial. Arch Neurol 1999; 56: 529-535.

7. Benbir G, Özekmekçi S, Apaydin H, Delil S, Erginöz E. A hospital-based study: Risk factors in development of motor complications in 555 Parkinson's patients on levodopa therapy. Clin Neurol Neurosur 2006; 108: 726-732.

8. Jankovic J. Motor fluctuations and dyskinesias in Parkinson's disease: Clinical manifestations. Mov Disord 2005; 20: S11-S16.

9. Garret MC, Rosas MJ, Simões F, Vieira S, Costa M. Does timing and dosage of levodopa modify druginduced dyskinesias and motor fluctuations? A retrospective analysis. Parkinsonism Relat Disord 1998; 4: 99-102.

10. Kostić VS, Marinković J, Svetel M, Stefanova E, Przedborski S. The effect of stage of Parkinson's disease at the onset of levodopa therapy on development of motor complications. Eur $\mathrm{J}$ Neurol 2002; 9: 9-14.
11. Kumar N, Van Gerpen JA, Bower JH, Ahlskog JE. Levodopa-dyskinesia incidence by age of Parkinson's disease onset. Mov Disord 2005; 20: 342-344.

12. Sharma JC, Ross IN, Rascol O, Brooks D. Relationship between weight, levodopa and dyskinesia: The significance of Levodopa dose per kilogram body weight. Eur J Neurol 2008; 15: 493-496.

13. Sharma JC, Bachmann CG, Linazasoro G. Classifying risk factors for dyskinesia in Parkinson's disease. Parkinsonism \& Related Disorders 2010; 16: 490497.

14. Schrag A, Quinn N. Dyskinesias and motor fluctuations in Parkinson's disease: A community-based study. Brain 2000; 123(11): 2297-2305.

15. Ku S, Glass GA. Age of Parkinson's disease onset as a predictor for the development of dyskinesia. Mov Disord 2010; 25: 1177-1182.16. DDD: Definition and general considerations. WHO Collaborating Centre for Drug Statistics Methodology [cited 2012 Nov 14]. Available from: http://www.whocc.no/ddd/definition_ and general consideral

16. Rossiter D, Ed. South African Medicines Formulary, 10th edn. Health and Medical Publishing Group; Cape Town; 2010; $p 641$.

17. Turner L, Ed. Daily Drug Use. 9th edn. The Tincture Press, Cape Town; 2010; $p 607$.

18. Thanvi B, Lo N, Robinson T. Levodopa-induced dyskinesia in Parkinson's disease: Clinical features, pathogenesis, prevention and treatment. Postgrad Med J 2007; 83: 384-388. 